\title{
PROPIEDADES PSICOMÉTRICAS DE LA ESCALA DE FELICIDAD SUBJETIVA (SHS) Y SU RELACIÓN CON EL ESTRÉS, LA SALUD PERCIBIDA Y EL APOYO SOCIAL EN PACIENTES CON CÁNCER DE MAMA
}

\author{
PSYCHOMETRIC PROPERTIES OF SUBJECTIVE HAPPINESS SCALE (SHS) AND ITS \\ RELATIONSHIP WITH STRESS, PERCEIVED HEALTH AND SOCIAL SUPPORT IN \\ PATIENTS WITH BREAST CANCER
}

\author{
Fresia Paloma Hernández Moreno y René Landero Hernández \\ Facultad de Psicología. Universidad Autónoma de Nuevo León. México
}

Resumen

El objetivo del estudio fue evaluar las propiedades psicométricas de la traducción al español de la Escala de Felicidad Subjetiva, y su relación con el estrés, la salud percibida y el apoyo social. Se utilizó el proceso de traducción inversa a fin de lograr una equivalencia conceptual de la escala. El estudio incluyó a una muestra no probabilística de 90 mujeres con cáncer de mama. Los resultados principales indican una estructura factorial de un factor explicando el $59,4 \%$ de la varianza. Se encontró relación entre felicidad y percepción de salud $(r=0,370, p=0,001)$, felicidad y estrés $(r=-0,462, p=0,001)$ y felicidad y apoyo social $(r=0,382, p=0,001)$. Se discuten los resultados encontrados y las limitaciones.

Palabras clave: Felicidad, apoyo social, estrés, salud percibida, cáncer de mama.
Abstract

The purpose of the study was to evaluate the psychometric properties of the Spanish translation of the Subjective Happiness Scale and its relation to stress, perceived health, and social support. Back translation procedure was used in order to achieve a conceptual equivalence of the scale. The study included a nonrandom sample of 90 women with breast cancer. The main results indicate a factorial structure of a factor explaining $59.4 \%$ of the variance. Was found a relation between happiness and perceived health $(r=0.370$, $\mathrm{p}=\mathbf{0 . 0 0 1})$, happiness and stress $(r=-0.462$, $p=0.001$ ) and finally happiness and social support $(r=0.382, p=0.001)$. The findings and limitations are discussed.

Key words: Happiness, social support, stress, perceived health, breast cancer.

\section{INTRODUCCIÓN}

Durante los últimos años, se ha vuelto importante en la investigación el tema de la felicidad subjetiva en diversas disciplinas, así como su influencia en diversos ámbitos del ser humano(1,2). Gran parte de lo que se ha hecho de investigación en ésta área, se ha enfocado en el bienestar subjetivo, el cual se considera una composición de afecto positivo, afecto negativo y satisfacción con la vida(3,4).

\section{Correspondencia:}

Fresia Paloma Hernández Moreno

Facultad de Psicología. Universidad Autónoma de Nuevo León

Av. Universidad s/n. Ciudad Universitaria, San Nicolás de los Garza, Nuevo León, C.P. 66451 México.

E-mail: fresiahdez@hotmail.com 
La felicidad se puede definir como el bienestar subjetivo, el cual se hace desde una evaluación personal, subjetiva y global y de su calidad cognitiva y afectiva de la vida de las personas ${ }^{(5,6)}$.

Una persona feliz, es aquella que cuenta con un temperamento positivo, que tiende a ver el lado favorable de las cosas y que no "rumia" de manera excesiva sobre los eventos malos, que cuenta con personas en quien confiar y amigos, además de que tiene recursos adecuados para orientarse a lograr sus metas significativas, y por consiguiente, cuenta con recursos para hacer frente de manera satisfactoria a las situaciones de estrés ${ }^{(7)}$.

La felicidad está dentro de lo que se consideran emociones positivas, que según Lykken ${ }^{(8)}$, los individuos son capaces de fortalecer de manera intencional su capacidad de experimentar y maximizar las emociones positivas, las cuales en diversos estudios se ha encontrado relación de estas con la mejora de la salud física, emocional y social ${ }^{(9)}$.

Las emociones positivas pueden estar centradas en el pasado, presente o futuro. En el futuro se encuentra el optimismo, la fe, la confianza y la esperanza; en el presente están la alegría, la tranquilidad, el flow (o experiencia óptima), el cual incluye la felicidad ${ }^{(1)}$ y sobre el pasado se incluye dentro de las emociones positivas la satisfacción, la complacencia, realización personal, el orgullo y la serenidad $^{(10)}$.

Los estados de ánimo y las emociones de las personas son el reflejo de las cosas que les suceden, en los cuales, cada persona hace juicios acerca de él y su vida en general. Por lo que existen un número de componentes divisibles del bienestar subjetivo, como pueden ser: la satisfacción con la vida, la cual se refiere al juicio global de la propia vida; la satisfacción con las áreas importantes de la vida, como puede ser por ejemplo, la satisfacción con el trabajo; el afecto positivo que se refiere a la experimentación de muchas emociones agradables y estados de ánimo positivos y por último los bajos niveles de afecto negativo, es decir el experimentar pocas emociones desagradables y estados de ánimo negativos ${ }^{(3)}$.

Un número considerable de estudios han indicado la influencia de la felicidad en la salud física, mental y en la creatividad de las personas, así como la infelicidad por el contrario, puede ser un factor que influya en la depresión e inclusive en el suicidio ${ }^{(11-15)}$.

Diener y Chan ${ }^{(16)}$, mencionan tres factores que frecuentemente se incluyen para enumerar las características de una buena vida: felicidad, salud y longevidad, lo cual como hemos visto, están sumamente relacionadas entre sí.

Además, existe gran evidencia de que el funcionamiento social y la felicidad están relacionados de manera importan$\mathrm{te}^{(17,18)}$. Como lo mencionan Jopp y $\operatorname{Rott}^{(19)}$, la salud física y el funcionamiento social se consideran determinantes de la felicidad en los adultos.

El apoyo social de la familia es comúnmente considerado como fuente primaria de la felicidad ${ }^{(20,21)}$. Las relaciones sociales se consideran como uno de los factores que favorecen de manera importante a la felicidad ya sea en adultos como en niños ${ }^{(22)}$. También, se ha encontrado que la calidad de las amistades que se tengan, independientemente del número de amigos con los que se cuentan, puede predecir la felicidad ${ }^{(23)}$.

Algunos estudios han reportado que las personas felices o con alto afecto positivo, manifiestan menores síntomas en el ámbito de la salud física y mental, así como en el funcionamiento social(20).

Las circunstancias de la vida, las metas y los valores personales pueden influir de manera importante en el bienestar subjeti$\mathrm{vo}^{(20,24,25)}$, ya sea una influencia positiva o 
negativa, de acuerdo a las circunstancias que la persona esté viviendo, así como la forma en la que evalúe dichos sucesos. Como lo mencionan Marans y Stimson ${ }^{(6)}$, la felicidad percibida puede verse afectada por diversos factores, los cuales pueden ir desde las perspectivas de empleo, las oportunidades para el crecimiento intelectual, la situación financiera y la seguridad personal.

Vivir con el diagnóstico de cáncer puede ser una fuente de estrés psicológico y social. En general, las mujeres con cáncer de mama pueden verse expuestas a situaciones que afecten su bienestar tanto físico como psicosocial. Ya que deben enfrentarse con el impacto producido por el diagnóstico y las consecuencias que esto trae, además de los efectos secundarios de los tratamientos, el cambio en su apariencia corporal, así como el miedo a la recurrencia, también en algunos casos las mujeres muestran un declive en su salud y un incremento en sus niveles de discapacidad física o hasta muerte ${ }^{(26)}$.

Algunas de las principales situaciones generadoras de estrés en el enfermo de cáncer son las emociones negativas (malestar emocional), el apoyo social percibido durante la enfermedad, es decir, que el apoyo social que reciban no sea el adecuado o suficiente y por último, la preocupación en cuanto al tratamiento y las molestias secundarias que este pueda generar ${ }^{(27)}$.

También, los problemas físicos asociados al cáncer pueden ser interpretados como fuente de estrés ${ }^{(28)}$. En un estudio cualitativo con 16 mujeres con cáncer de mama que habían recibido mastectomía (total o parcial), radioterapia y/o quimioterapia y que se encontraban entre uno y cinco años post-diagnóstico, el cual tuvo el objetivo de conocer los eventos estresantes en estas pacientes, se encontró que los principales factores eran: la alteración de su cuerpo debido a los diversos tratamientos, la recurrencia de pensamientos y sentimientos experimentados en relación a su condición de salud, los conflictos que se generaron en sus áreas familiares y sociales, así como el aspecto laboral, debido a las alteraciones experimentadas en su capacidad productiva en ésta área ${ }^{(29)}$.

La mayoría de los estudios que se han hecho respecto a la felicidad subjetiva han sido en población general $\left.\right|^{(2,6,7,11)}$, población universitaria ${ }^{(14,30)}$, en niños ${ }^{(22)}$ y en adultos mayores $^{(16,17)}$, por lo que se hace importante el estudiar esta variable desde la perspectiva de los pacientes con enfermedades crónicas, en este caso en pacientes con cáncer de mama.

Por lo anterior, el presente estudio tuvo como objetivo el analizar la relación de la felicidad con el estrés percibido, el apoyo social y la salud percibida, así como la comparación de dos grupos respecto a la felicidad subjetiva entre quienes se perciben con menor y mayor salud, validando de forma previa la estructura factorial de la escala de Felicidad Subjetiva de Sonja Lyubomirsky y Lepper(31), en una muestra de mujeres con cáncer de mama.

\section{MÉTODO}

\section{Participantes}

Se utilizó un muestreo no probabilístico por conveniencia. Los criterios de inclusión fueron el tener cáncer de mama y haber acudido a consulta médica en el Centro Universitario Contra el Cáncer de la ciudad de Monterrey, Nuevo León, México y al Hospital de Gineco-Pediatría No. 2 del IMSS en la ciudad de Los Mochis, Sinaloa, México, además que fueran mayores de 25 años y que contaran con habilidades de lecto-escritura para el llenado de los instrumentos. Como criterios de exclusión se fijaron el no estarse atendiendo en los hospitales antes men- 
cionados, contar con alguna enfermedad discapacitante a nivel funcional que impidiera el llenado de los instrumentos o el negarse a participar en el estudio. La muestra se conformó por un total de 90 pacientes, de las cuales el 55,6\% fueron de la Ciudad de Los Mochis, Sinaloa y el resto de Monterrey, Nuevo León.

\section{Procedimiento}

La muestra se obtuvo acudiendo a los hospitales que se mencionaron con anterioridad. Los cuestionarios fueron de forma auto aplicable y antes del llenado de los mismos se procedió a firmar el consentimiento informado de la investigación en el cual se les garantizaba la confidencialidad, así como el cumplimiento de sus derechos como participante, de acuerdo a los lineamientos de investigación de la American Psychological Association ${ }^{(32)}$. Después de esto se procedió a la cumplimentación de los instrumentos por parte de las participantes.

\section{Instrumentos}

\section{Escala de Estrés Percibido}

Para medir el estrés se utilizó la Escala de Estrés Percibido (Perceived Stress Scale, PSS) elaborada por Cohen et al. ${ }^{(33)}$ para evaluar el grado en que las situaciones de la vida son valoradas como estresantes, obteniendo un alfa de Cronbach de 0,75. En este estudio se utilizó la adaptación cultural realizada en México por González y Landero ${ }^{(34)}$, la cual cuenta con 14 ítems y una consistencia interna $(\alpha=0,83)$, además de confirmar la estructura factorial de la escala original, utilizando un análisis factorial confirmatorio. La puntuación va de nunca $-0-$ a muy a menudo $-4-$. Invirtiéndose la puntuación en los ítems: $4,5,6,7,9,10$ y 13. La escala cuenta con un rango de puntuaciones posibles que van de 0 a 56. La mayor puntuación corresponde a mayor estrés percibido.

\section{EORTC QLQ-C30 3.0 Calidad de Vida}

Para la medición de la calidad de vida se utilizó el instrumento QLQ-C30 3.0 que fue elaborado por el grupo de Calidad de Vida de la Organización Europea para la Investigación y Tratamiento del Cáncer (EORTC) la cual cuenta con una confiabilidad de entre 0,70 y $0,87^{(35)}$. Incluye cinco escalas funcionales: funcionamiento físico, rol, emocional, social y cognitivo; tres escalas de síntomas: fatiga, dolor, y náusea/ vómitos y una escala global de salud/calidad de vida. Además de ítems individuales que evalúan síntomas adicionales de disnea, pérdida de apetito, insomnio, estreñimiento y diarrea, y el impacto financiero. Consta de 30 preguntas las cuales cuentan con un formato de respuesta tipo Likert de cuatro puntos, excepto en la escala global que tiene siete. Todas las escalas e ítems individuales se convierten en una puntuación de 0 a 100, siendo este el rango de puntuaciones posibles para la escala. De esta escala solo se consideró para este estudio la escala global de salud/calidad de vida.

\section{Cuestionario de Apoyo social}

Para la evaluación del apoyo social se utilizó el cuestionario MOS de apoyo social fue elaborado por Sherbourne y Stewart ${ }^{(36)}$, para evaluar el apoyo social que perciben. En este estudio se utilizó la validación en Argentina por Espínola-Rodríguez y HéctorCarmelo ${ }^{(37)}$, la cual mostró una consistencia interna de 0,91 según el alfa de Cronbach, la cual cuenta con 12 ítems, donde el primer ítem evalúa el número de personas cercanas (red). La puntuación va de nunca -1 - a siempre -5 - Teniendo un rango de puntuaciones posibles que van de 1 a 60 . La mayor puntuación corresponde a mayor apoyo social percibido. 
Escala de felicidad subjetiva (SHS)

Para la variable Felicidad se utilizó la Escala de Felicidad subjetiva (SHS), elaborada por Lyubomirsky y Lepper ${ }^{(31)}$ para evaluar la felicidad percibida. En este estudio se utilizó, la versión en español traducida por el método back translation para la investigación de Hernández ${ }^{(38)}$, donde se obtuvo un alfa de Cronbach de 0,73 , que se considera adecuada, ya que se compone de 4 ítems. Teniendo un rango de puntuaciones posibles de 4 a 28. La traducción se cotejó con la versión española de Extremera et al. ${ }^{(39)}$.

Los resultados han indicado que el SHS tiene una adecuada consistencia interna, por medio de los test-retest y la correlación han sugerido una buena confiabilidad, y los estudios de validez de constructo sobre la validez convergente y discriminante han confirmado el uso de esta escala para medir el constructo de la felicidad subjetiva.

\section{Análisis estadísticos}

Teniendo recolectada la muestra, se procedió a capturar los datos obtenidos en el programa estadístico SPSS 18, posteriormente se analizó la consistencia interna de los instrumentos de evaluación, la cual se obtuvo mediante el coeficiente alfa de Cronbach. Se realizó un análisis factorial exploratorio por componentes principales. Las correlaciones entre las variables se calcularon mediante el coeficiente producto-momento de Pearson, además, se realizó la comparación de dos grupos mediante la t de Student para muestras independientes. El ajuste de la distribución a una curva normal se contrastó mediante la prueba de Kolmogorov-Smirnov.

\section{RESULTADOS}

\section{Descripción de la muestra}

El total de la muestra fue de 90 participantes, todas mujeres con cáncer de mama, de las cuales el 55,6\%, fueron de la ciudad de Los Mochis Sinaloa y el resto de la ciudad de Monterrey Nuevo León. La media de edad fue de 49,5 (DT=9,9), con un rango entre 36 y 76 años. En relación a los estudios, se obtuvo una media de años de $9(\mathrm{DT}=4,2)$, lo cual corresponde al nivel del secundaria terminada. En cuanto al estado civil, 12 (13,3\%) dijeron estar solteras, $52(57,8 \%)$ casadas, $7(7,8 \%)$ divorciadas, $10(11,1 \%)$ separadas, $4(4,4 \%)$ en unión libre y $5(5,6 \%)$ viudas. La media de tiempo de diagnóstico de las pacientes en la muestra total fue de 19 meses.

En cuanto a aspectos clínicos de las pacientes, el $41,1 \%$ se encontraba recibiendo quimioterapia, el $25,6 \%$ se encontraba recibiendo quimioterapia oral, el 2,2\% se encontraba en radioterapia, mientras que el $6,7 \%$ recibía quimioterapia oral e intravenosa, el $1 \%$ quimioterapia oral y radioterapia y el resto no se encontraban recibiendo ningún tratamiento activo para el cáncer de mama.

La consistencia interna, que se evaluó a través del coeficiente alfa de Cronbach, para cada instrumento utilizado en esta investigación, se muestran en la Tabla 1 , junto con los análisis descriptivos de cada variable.

Se realizó el análisis factorial de la Escala de Felicidad Subjetiva, en el cual el valor de $\operatorname{KMO}(0,68)$ es aceptable y la prueba de esfericidad fue significativa $(p=0,001)$. Se realizó mediante el método de componentes principales quedando 1 factor. En la figura 1 puede observarse el gráfico de sedimentación. Este único factor explica el $59,4 \%$ de la varianza. La saturación de cada ítem del análisis factorial exploratorio se muestra en la tabla 2.

Para conocer la relación entre las variables se realizó el cálculo mediante el coeficiente producto momento de Pearson, los resultados se muestran en la tabla 3. Como puede observarse, se encontró relación entre felicidad y percepción de 
Tabla 1. Análisis descriptivos de las variables y coeficiente del alfa de Cronbach.

\begin{tabular}{|lcccc|}
\hline & Felicidad & Salud Percibida & Estrés & Apoyo Social \\
\hline N & 90 & 89 & 90 & 90 \\
Alfa de Cronbach & 0,75 & 0,93 & 0,70 & 0,90 \\
Media & 19,5 & 5,4 & 24,5 & 44,5 \\
Mediana & 19,5 & 5,5 & 25 & 46 \\
Desviación Estándar & 5,5 & 1,1 & 6,5 & 9,4 \\
\hline
\end{tabular}

Figura 1. Gráfico de sedimentación para la Escala de Felicidad Subjetiva (SHS)

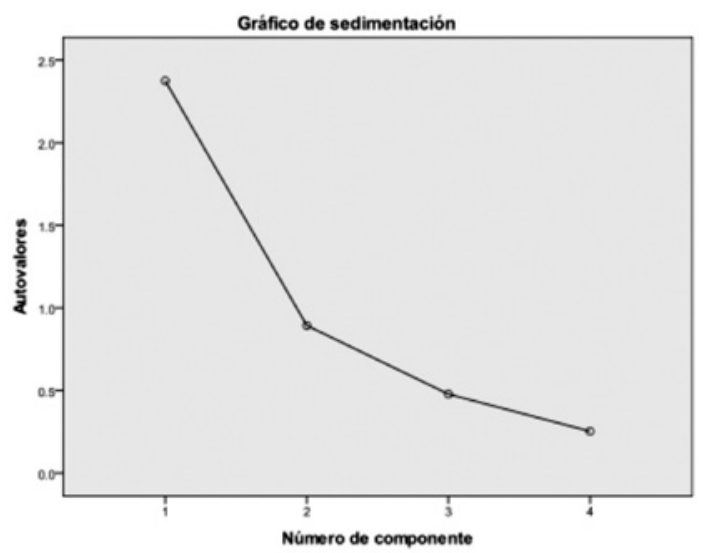

Tabla 2. Estructura factorial de la SHS Escala de Felicidad Subjetiva mediante el método de componentes principales.

\begin{tabular}{|l|c|}
\hline \multicolumn{1}{|c|}{ Ítems } & Saturación \\
\hline 1. En general me considero & 0,840 \\
\hline 2. En comparación con la mayoría de mis compañeros me considero & 0,888 \\
\hline 3. Algunas personas son generalmente son muy felices & 0,833 \\
\hline 4. Algunas personas son generalmente no muy felices & 0,434 \\
\hline
\end{tabular}

salud ( $r=0,370, p=0,001)$, felicidad y estrés $(r=-0,462, p=0,001)$ y felicidad y apoyo social ( $r=0,382, p=0,001)$. También se encontró relación negativa entre el estrés y la percepción de salud $(r=-0,333, p=0,001)$ y finalmente entre apoyo social y estrés también se encontró una relación negativa $(r=-0,265, p=0,012)$. No se encontró relación significativa entre el apoyo social y la percepción de salud.

Finalmente, se evaluaron las diferencias respecto a las pacientes que se percibían con mayor salud (grupo 2) y con menor salud (grupo 1) en cuanto a la felicidad, 
Tabla 3. Correlaciones entre las variables.

\begin{tabular}{lcccc}
\hline & Felicidad & $\begin{array}{c}\text { Percepción de } \\
\text { salud }\end{array}$ & Estrés & Apoyo social \\
\hline Felicidad & 1 & & & \\
Percepción de salud & $0,370^{* *}$ & 1 & 1 & \\
Estrés & $-0,462^{* *}$ & $-0,333$ & $-0,265^{*}$ & 1 \\
Apoyo social & $0,382^{* *}$ & 0,323 & \\
\hline
\end{tabular}

Análisis de correlación mediante el coeficiente de Pearson.

${ }^{*} \mathrm{p}<0,05,{ }^{* *} \mathrm{p}<0,01,{ }^{* * *} \mathrm{p}<0,001$.

mediante el análisis de T de Student. La homogeneidad de varianza se evaluó mediante la prueba de Levene en la cual la variable felicidad no resultó significativa ( $\mathrm{F}=0,228, \mathrm{p}=0,634)$.

En cuanto a diferencias entre los grupos de quienes se percibían con mayor y menor salud, respecto a la felicidad, se encontró diferencia significativa negativa ( $T=-3,207, g l=87, p=0,002)$, es decir, quienes se perciben con mayor salud manifiestan estar más felices.

\section{DISCUSIÓN/CONCLUSIONES}

Al comparar los resultados del estudio con los reportados por Extremera et al. ${ }^{(39)}$, quienes elaboraron la versión Española de la SHS, se puede observar que la varianza explicada de la presente investigación en el análisis factorial exploratorio es inferior a la reportada por los autores, quienes encontraron un $63,5 \%$ de varianza explicada, ya que con la muestra de este estudio la varianza fue de 59,4\%. Por otra parte en una muestra de jóvenes universitarios y adultos de la ciudad de Córdoba, Argentina, se encontró una varianza de $57,38 \%{ }^{(40)}$, lo cual está por debajo de lo encontrado en nuestro estudio. Lo anterior nos muestra que la escala SHS es una medida útil y reconocida en muchos países de Latinoamérica y el mundo, y es además un instrumento corto para investigadores que tienen una batería muy extensa y esto ayuda a no extender aún más la batería, y lo más importante, que es una medida fiable y válida para ser utilizada en muestras de habla castellana.

En el presente estudio se encontró una relación positiva entre la salud y la felicidad percibida de las pacientes, lo cual nos indica, que entre mejor sea la percepción de salud, tendrán mayor felicidad percibida. Siendo estos resultados consistentes con los encontrados por Gerstenbluth et al. ${ }^{(12)}$, quienes afirman que se puede considerar al estado de salud como uno de los principales determinantes de la felicidad, ya que el hecho de tener una buena salud, incrementa considerablemente la felicidad. Como lo mencionan Pressman y Cohen $^{(41)}$, que al aumentar la gravedad de la enfermedad, el afecto positivo, incluyendo la felicidad, disminuyen. También Moyano y $\operatorname{Ramos}^{(11)}$, en una muestra conformada por trabajadores y estudiantes, encontraron una relación significativa entre la autoevaluación de salud y la felicidad. Esto también concuerda con los resultados obtenidos por Blanchflower y Oswald ${ }^{(42)}$, en su investigación con pacientes hipertensos quienes concluyen que aquellos con menores problemas de hipertensión son quienes reportan los niveles más altos de satisfacción con la vida. Como se trata de una relación bivariada, también se puede considerar el efecto de la felicidad sobre la salud, es decir, quienes experimentan emociones positivas, pueden ayudar a 
incrementar tanto la salud física, como mental, como lo mencionan Lyubomirsky et al. ${ }^{(20)}$, que las personas felices o que presentan alto afecto positivo, reportan menos sintomatología en el ámbito de la salud física y mental. Esto además se corroboró en la presente investigación, mediante el análisis de comparación de grupos, respecto a quienes se percibían con mayor y menor salud respecto a la felicidad percibi$\mathrm{da}$, demostrando que quienes se perciben más sanos, manifiestan estar más felices. Dando lo anterior, importante sustento en cuanto a la relación de la percepción de salud y su influencia en la felicidad de la población general, así como también en la población clínica como se vio en esta muestra.

Se encontró una relación negativa entre el estrés percibido y la felicidad, ya que en este tipo de enfermedades puede verse afectada la seguridad personal de las pacientes, se enfrentan a modificaciones en aspectos importantes de su vida, como puede ser el rol familiar, el trabajo y los aspectos sociales, además de la preocupación de enfrentarse al tratamiento y las molestias secundarias que este pueda generar. La relación de estas dos variables puede explicarse por la situación vital de las pacientes con cáncer de mama ya que se enfrentan a una enfermedad crónica que merma de manera importante su calidad de vida, ya que como mencionan Reich y Remor $^{(26)}$, vivir con el diagnóstico de cáncer, puede ser una fuente de estrés psicológico y social. En general las mujeres con cáncer de mama pueden verse expuestas a situaciones que afecten su bienestar tanto físico como psicosocial.

Se corroboró la relación entre el estrés y la salud percibida, hallazgo que es compartido con otros trabajos de investigación con resultados similares como el de Juárez y Landero ${ }^{(43)}$, en una muestra mexicana de mujeres con cáncer de mama, demostrando que los estados emocionales pueden influir de manera directa en el funcionamiento fisiológico de las pacientes con cáncer de mama. Esto consistente también con los resultados reportados por Silva y Dos Santos ${ }^{(29)}$, en el que se encontró que los principales factores de estrés en las pacientes con cáncer de mama eran, la alteración de su cuerpo debido a los diversos tratamientos, la recurrencia de pensamientos y sentimientos experimentados en relación a su condición de salud, los conflictos que se generaron en sus áreas familiares y sociales, así como el aspecto laboral, debido a las alteraciones experimentadas en su capacidad productiva en ésta área.

Durante los últimos años, el interés por los factores psicológicos que mejoran la salud y el bienestar, como es el caso de la psicología positiva la cual se centra en variables que contribuyen en el crecimiento personal, funcionando como factores de protección o amortiguadores ante las situaciones difíciles que se puedan presentar en la vida de las pacientes, como lo es el apoyo social percibido, ya que funciona como amortiguador ante las situaciones de estrés, así, entre más y mejor apoyo social perciban, menores serán los niveles de estrés reportados, además de estar directamente relacionado con la felicidad percibida, como se ha confirmado en este estudio. Siendo estos resultados congruentes con diversos estudios ${ }^{(7,18,20)}$, quienes afirman que tanto el tener amigos, así como la calidad de esas amistades, potencian la felicidad, así como también, que las personas más felices pasan más tiempo conviviendo con sus amigos y tienen un mejor funcionamiento social. Esto también consistente con lo encontrado por SchmidBuchi et al. ${ }^{(4)}$, en una muestra de mujeres recién diagnosticadas con cáncer de mama, quienes concluyen que la falta de apoyo social, los conflictos en sus relaciones personales se asociaron con más necesidades de apoyo y cuidados por parte del 
equipo de asistencia médica, además, son quienes manifestaban más síntomas como fatiga, sofocos y cambio en su apariencia corporal. Además las pacientes con más problemas psicológicos solicitaban más atención médica.

Finalmente, no se corroboró una relación significativa entre el apoyo social percibido y la salud percibida. Esto se puede explicar mediante la relación que existe entre el apoyo social y el estrés y la relación entre la salud percibida y el estrés, ya que puede estar actuando de forma indirecta por medio de las asociaciones antes mencionadas.

A partir de los resultados del presente trabajo, se pretende proveer información necesaria a los profesionales de la salud mental, para la elaboración de programas de acción y prevención en el tratamiento psicológico de las pacientes con cáncer de mama. Haciendo hincapié en los recursos psicológicos que mejoran la salud y potencian la felicidad, todo con el fin de contribuir en el desarrollo del bienestar integral de las pacientes. Se recomienda seguir investigando en los aspectos psicológicos que mejoran la salud y la felicidad de pacientes con este tipo de padecimientos, así como indagar de manera más específica en la calidad y cantidad de apoyo social que reciben, así como también sería importante el analizar los niveles de felicidad y percepción de salud de acuerdo a la condición de estado civil.

\section{REFERENCIAS BIBLIOGRÁFICAS}

1. Seligman MEP. La auténtica felicidad. España: Vergara, 2003.

2. Vera-Villarroel P, Celis-Atenas K, CórdobaRubio N. Evaluación de la felicidad: análisis psicométrico de la Escala de Felicidad Subjetiva en población chilena. Ter Psicol 2011; 29:127-33.

3. Diener E. Subjetive well-being: The science of happiness and a proposal for a
National Index. Am Psychol 2000;55:3443. Doi: 10.1037/110003-66x.55.1.34.

4. Bartels M, Boomsma DI. Born to be happy? The etiology of subjective well-being. Behav Genet 2009; 39:605-15.

5. Diener E, Suh E, Lucas R, Smith H. Subjetive well-being: three Decades of Progress. Psychol Bull 1999;125:276-302. Doi:10.1037//0033-2909.125.2.276

6. Marans RW, Stimson RJ, editors. Investigating quality of urban life. Theory, methods and empirical research. Netherlands: Springer Publishing, 2011.

7. Diener E, Seligman M. Very happy people. Psychol Sci 2002;13:81-4. Doi: 10.111/1467-92-80.

8. Lyken D. The nature and nurture of joy and contentment. New York: St. Martins Griffin, 2000.

9. Fredrickson B. Positive emotions. En: Zinder C, López S, editors. Handbook of positive Psychology. New York: Oxford University Press, 2000. p.120-34.

10. Seligman M, Csikszentmihalyi M. Positive Psychology: An introduction. Am Psychol 2000;55:5-14.Doi: 10.1037//0003066X.55.1.5

11. Moyano E, Ramos N. Bienestar subjetivo: midiendo satisfacción vital, felicidad y salud en población chilena de la región Maule. Universum 2007;22:173-93.

12. Gerstenbluth M, Rossi M, Triunfo P. Felicidad y salud: una aproximación al bienestar en el Río de la Plata. Estud Econ 2008;35:65-78.

13. Koopmans T, Geleijnse J, Zitman F, Giltay E. Effects of happiness on all-cause mortality during 15 years of follow-up: The arnhem elderly study. J Happiness Stud 2010;11:11324. Doi:10.1007/s10902-008-9127-0

14. Mustaca A, Kamenetzky G, Vera-Villarroel P. Relaciones entre variables positivas $y$ negativas en una muestra de estudiantes argentinos. Rev Argent Clin Psicol 2010;19:227-35.

15. Piqueras JA, Kuhne W, Vera-Villarroel $P$, van Straten A, Cuijpers P. Happiness and 
health behaviours in chilean college students: A cross-sectional survey. BMC Public Health 2011;11:443-52. Doi: 10.1186/1471-2458-11-443.

16. Diener E, Chan MY. Happy people live longer: subjetive well-being contributes to health and longevity. Appl Psychol Health Well-Being 2011;3:1-43. Doi:10.1111/ j.1758-0854.2010.01045.x

17. Bishop AJ, Martin P, Poon L. Happiness and congruence in older adulthood: A structural model of life satisfaction. Anging Ment Health 2006;10:445-53. Doi:10.1080/13607860600638388

18. Cacciopo JT, Hawkley LC, Kalil A, Hughes ME, Waite L, Thisted RA. Happiness and the invisible threads of social connection: The Chicago health aging and social relations study. En: Eid M, Larsen R, editors. The Science of subjective well-being. New York: Guildford Press, 2008. p.195-219.

19. Jopp D, Rott C. Adaptation in very old age: Exploring the role of resources, beliefs and attitudes for centenarian's happiness. Psychol Aging 2008; 21:266-80. Doi: 10.1037/0882-7974.21.2.266.

20. Lyubomirsky S, King L, Diener E. The benefits of frequent positive affect: Does happiness lead to success? Psychol Bull 2005;131:803-55. Doi:10.1037/00332909.131.6.803

21. North RJ, Holahan CJ, Moos RH, Cronkite RC. Family support, family income and happiness: A 10-year perspective. J Fam Psychol 2008;22:475-83.

22. Holder MD, Klassen A. Temperament and Happiness in children. J Happiness Stud 2010;11: 419-39. Doi:10.1037/08933200.22.3.475

23. Demir M, Weitekamp LA. "I am so happy cause today I found my friend: friendship and personality as predictors of happiness". J Happiness Stud 2006;8:181-211. Doi:10.1007/s10902-006-9012-7.

24. Vinaccia S, Quiceno M. Calidad de vida relacionada con la salud y factores psicológicos: un estudio desde la enfermedad pulmonar obstructiva crónica- EPOC. Ter Psicol 2011; 29:65-75.

25. Urzúa A, Pavlov R, Cortés R, Pino V. Factores psicosociales relacionados con la calidad de vida en salud en pacientes hemodializados. Ter Psicol 2011;29:135-40.

26. Reich M, Remor E. Variables psicosociales asociadas con calidad de vida relacionada con la salud en mujeres con cáncer de mama post-cirugía: una revisión sistemática. Ciencias Psicológicas 2010;4:177-221.

27. Pulgar MA, Garrido S, Muela JA, Reyes GA. Validación de un inventario para la medida del estrés percibido y las estrategias de afrontamiento en enfermos de cáncer (ISEAC). Psicooncología 2009;6:167-90.

28. Constanzo EE, Lutgendort SK, Mattes ML, Trehan S, Robinson CB, Tewfick F, Roman SL. Adjusting to life after treatment: distress and quality of life following treatment for breast cancer. Br J Cancer 2007;97:162531. Doi: 10.1038/sj.bcg.6604091

29. Silva G, Dos Santos MA. Factores estresantes del post-tratamiento del cáncer de mama: un enfoque cualitativo. Rev LatinoAm Enfermagem 2010;18:1-8.

30. Omar A, Paris L, Aguiar de Souza M, Almeida da Silva $S$, \& Del Pino- Piña, R. Validación del inventario de bienestar subjetivo con muestras de jóvenes y adolescentes argentinos, brasileños y mexicanos. Suma Psicol 2009;16:69-84.

31. Lyubomirsky S, Lepper H. A measure of subjetive happiness: preliminary reliability and construct validation. Soc Indic Res 1999;46:137-55. Doi: 10.1023/A:1006824100041

32. American Psychological Association [APA]. Principios Éticos del Psicólogo y Código de Conducta, 2010.En línea [Acceso el 10 de marzo de 2014] Disponible en: http://www.apa.org/ethics/code/index. aspx.

33. Cohen S, Kamarck T, Mermelstein R. A global measure of perceived stress. J Health Soc Behav 1983;24:385-96.Doi: $10.2307 / 2136404$ 
34. González M, Landero R. Factor Structure of the Perceived Stress Scale (PSS) in a sample from Mexico. Span J Psychol 2007;10:199-206.

35. Fayers $\mathrm{P}$, Aaronson N, Bjordal K, Groenvold M, Curran D, Bottomley A. $3^{\text {rd }}$ ed. The EORTC QLQ-C30 Scoring Manual. Bruselas: European Organization for Research and Treatment of Cancer, 2001.

36. Sherbourne CD, Stewart AL. The MOS Social Support Survey. Soc Sci Med 1991; 32:705-14. Doi10.1016/02779536(91)90150-B

37. Espínola-Rodríguez S, Carmelo E. Validación Argentina del cuestionario MOS de Apoyo Social Percibido. Psicodebate Psicología Cultura Sociedad 2010;7:155-68.

38. Hernández FP. Estudio comparativo de variables psicosociales relacionadas con la calidad de vida en mujeres con cáncer de mama [Tesis]. Monterrey: Universidad Autónoma de Nuevo León. Facultad de Psicología: 2012.

39. Extremera N, Fernández P, González V, Cabello R. Una validación preliminar de la versión española de la Subjective
Happiness Scale. En: Fernández-Berrocal $\mathrm{P}$, editor. Avances en el estudio de la inteligencia emocional. España: Fundación Marcelino Botín, 2009.p.39-44.

40. Ortíz MV, Gancedo KM, Reyna C. Propiedades psicométricas de la Escala de Felicidad Subjetiva en jóvenes y adultos de la ciudad de Córdoba, Argentina. Suma Psicol 2013;20:45-56.

41. Pressman SD, Cohen S. Does positive affect influence health? Psychol Bull 2005;131:925-71. Doi: 10.1037/00332909.131.6.925

42. Blanchflower DG, Oswald AJ. Hypertension and happiness across nations. J Health Econ 2008;27:218-33. Doi:10.1016/j. jhealeco.2007.06.002

43. Juárez D, Landero R. Variables psicosociales y salud en mujeres con cáncer de mama. Summa Psicol-UST 2009;6:79-88.

44. Schmid-Buchi S, Halfers RJG, Muller M, Dassen T, Van der Borne B. Factors associated with supportive care needs of patients under treatment for breast cancer. Eur J Oncol Nurs 2013;17:22-9. Doi:10.1016/j. ejon.2012.02.003 
